\title{
Motivational Emotion Generation and Behavior Selection based on Emotional Experiences for Social Robots
}

\author{
Won Hyong Lee ${ }^{1}$, Jeong Woo Park ${ }^{1}$, Woo Hyun Kim ${ }^{1}$, Hui Sung Lee ${ }^{2}$, \\ Dong-Soo Kwon ${ }^{1}$, and Myung Jin Chung ${ }^{1}$ \\ ${ }^{1}$ Korea Advanced Institute of Science and Technology (KAIST) \\ ${ }^{2}$ Hyundai-KIA MOTORS \\ leestation@kaist.ac.kr
}

\begin{abstract}
As the number of social and psychological service robots increase, many robots which have emotions have been developed. In order to implement an emotion generation model, the authors of this paper suggest to employ the unified motivation theory which is proposed by Higgins. In addition, a behavior selection model based on emotional experience is suggested. A joint attention scenario is suggested in which a human and a robot play a 2048 game together with a follow-up interaction. A preliminary pilot test was conducted to examine the proposed method and it performed properly as intended. Finally, further experiment plans and expectations are discussed.
\end{abstract}

Keywords: Motivation, Emotion, Attention, Emotional Experiences, Social Robot

\section{Introduction}

People expect that robots will be partners coexisting with human beings and assist human not only physically but also psychologically. According to World Robot Declaration which was issued by the international robot fair 2004, one of the expectation for next-generation robots is that robots will be social members in our human society. After 10 years later, nowadays, robots can be found easily in our daily lives and they are being used in different and various social areas such as supporting the elderly's emotional communication, helping autism treatment, and so on. Social Robotics, is becoming one of the very important topics in the robotic field [1][2].

In order for robots to supply with psychological and sociable assistant, robots are expected to show emotional expressions. Researchers have been developed emotional robots, for examples, KISMET, AIBO, KOBIAN, HRP-4C, KaMERo, etc [3-7]. Recently, Pepper and Jibo were announced that they will be released in the near future, and got lots of people's expectation and interest. The market of social and emotional robots is getting bigger.

To design an emotional robot, the meaning of emotions for a robot needs to be firstly defined and a model for robot's emotion generation should be established. 
There are several computational emotion models which already have been suggested [3][8-10], however, in this paper the authors in this paper will suggest a new perspective based on the generation of the emotional state through motivations as suggested by the unified motivation theory. This is a novel approach respect to the current computational models of emotions. In addition, we suggest a robot's behavior selection model based on emotional memory which has been experienced through interaction between a human and a robot.

Motivation and emotion imply social attention [11]. If a robot is motivated in a situation, it will concentrate and consume more resources on that situation, i.e. it shows more attention to the situation than other situations. Then, the robot experiences higher emotional intensity according to its motivational engagement level and situational results [12]. Reflecting this concept to the human and robot interaction scenario, a robot shows more attention to social situation or human companions which are more emotional, and the attention and emotion affect robot's social states such as intimacy, loyalty, and so on, so that the robot is able to properly behave based on the social states.

\section{Proposed Method}

\subsection{Motivational Emotion Generation}

The definition of emotion has not yet definitely defined. The most famous psychologist, Plutchik defined that every emotion is reaction to stimuli [13]. Although people sometimes seem to feel happy or angry in long time without any clear reason, psychologists have called it mood, global affect, or temperament, which are usually distinguished from emotion. In general, emotion is defined as temporal experience and reaction to certain events [14].

According to Plutchik's definition, the basic process of emotion generation is cognitive appraisal process, and the evaluated emotions raise physical changes and emotional reaction. As the cognitive appraisal theories describe motivation is a precursor of emotion, one crucial function of emotional experiences is to signal or provide feedback about motivational states [15].

Following the above concept, emotions of a robot can be defined as feedback signals from a robot's internal motivational states and they are caused by events. Therefore, motivational states and relations between motivation and emotion need to be organized as previous step for emotion generation.

Motivation is like a source of behaviors, and it makes goals and encourages acting. Since motivations, however, cannot be observed directly, several theories exist to explain them: considering motivation as all-purpose energy, motivation as approaching or avoiding something, or motivation as preferences directing choices, etc. In order to integrate motivational theories, Tory Higgins has proposed a unified motivation theory which describes motivation as a concept of three ways of being effective: value, truth, and control effectiveness [15]. Value effectiveness is about having desired result, truth effectiveness is about establishing what's real, and control effectiveness is about managing what happens. Following this theory, in a situation that at least one of 
the effectiveness occurs, robots are motivated and engaged, then, therefore they pay attention and feel higher level of emotions to the situation. This motivational attention and emotion cause dynamic changes in robot's behavior decision condition later on.

According to the theory, if an effectiveness is high (success), then we experience pleasant emotions, such as cheerful, quiescent, confidence, vigorous, etc. If the effectiveness is low (failure), in other words, if we fail to have the desired result, we experience painful emotions such as dejected, agitated, confused, Incompetent, etc. The relations between emotions and the motivational states which are value, truth, and control effectiveness are summarized up in Table 1 . These relations are applied to the robot's emotion generation model.

Table 1. Relation between effectiveness and emtion in accordance with failure and success

\begin{tabular}{|c|c|c|}
\hline & Failure (Pain) & Success (Pleasure) \\
\hline Value Effectiveness & Dejected, Agitated & Cheerful, Quiescent \\
\hline Truth Effectiveness & Confused, Surprised & Confidence, Sure \\
\hline Control Effectiveness & Powerless, Incompetent & Vigorous, Officious \\
\hline
\end{tabular}

Moreover, among pleasant/painful emotions, the emotions can be distinguished by levels of arousal factor which is a basic element of emotion and Regulatory focus theory is applied to evaluate them [12]. According to the theory, the level of arousal is determined by regulatory focuses: promotion focus and prevention focus. There are three elements that determine the regulatory focus. Those are Hedonic properties (positive/negative outcome situations), Standards (ideals or oughts), and Need satisfaction. Standards are given in the context of situation and interaction, and need satisfaction is depending on robot's current conditions such as thirst, hunger, fatigue, etc. With these elements, promotion focus is determined by gain/non-gain situation, strong ideals, and nurturance needs, and prevention focus is determined by non-loss/loss situation, security needs, and strong oughts. After a regulatory focus is set, the level of arousal factor can be differentiated by the type of regulatory focus. The relations between the regulatory focus and the emotional elements in accordance with success/failure of effectiveness are summarized in Table 2.

Table 2. Relations between the regulatory focus and the emotional elements(valence, arousal) in accordance with success and failure of effectiveness

\begin{tabular}{|c|c|c|}
\hline & Failure & Success (Pleasure) \\
\hline Promotion Focus & Pain, Low Arousal & Pleasure, High Arousal \\
\hline Prevention Focus & Pain, High Arousal & Pleasure, Low Arousal \\
\hline
\end{tabular}

Through these procedures, we can evaluate a robot's two emotional elements, valence (pleasure/pain) and arousal, from motivational states. With these two elements, we can use two-dimensional emotion space that has two axes: valence axis and arousal axis. Let a robot's emotion state be a vector $\mathbf{a}[n]$ in the emotion space at step $n$ and let elements of the vector at step $n$ be valence $[n]$ and arousal $[n]$. Thus, the emotion state of a robot at step $n$ is defined as 


$$
\mathbf{a}[n]=(\text { valence }[n], \text { arousal }[n])
$$

where valence $[n] \in[-1,1]$ and 1 denotes maximum of pleasure, -1 denotes maximum of pain, and arousal $[n] \in[-1,1]$ and 1 denotes maximum level of arousal, -1 denotes minimum level of arousal.

To evaluate specific emotion type $i$ and its intensity $e_{i}[n]$, we can employ Russell's Circumplex model [16]. The model indicates where the 28 target emotions are distributed in the two-dimensional emotion space. Let the position vector of the target emotion be $\mathbf{t}_{i}=\left(\right.$ valence $_{i}$, $\left._{\text {rousal }}\right)$ where $i$ is an emotion type among 28 target emotions described in Circumplex model. The number of emotion types may narrow down to the emotions that are mentioned in Table 1 . Then, the distance between $\mathbf{a}[n]$ and $\mathbf{t}_{i}$ determines the intensity of specific emotion $e_{i}[n]$ by using Gaussian shape function as

$$
e_{i}[n]=\exp \left(-\left(\frac{\left\|\mathbf{t}_{i}-\mathbf{a}[n]\right\|}{2 \sigma^{2}}\right)\right)
$$

where $\sigma$ is a parameter for the narrowness of the Gaussian shape and it is determined by developers. Furthermore, we may consider the specific emotion $e_{i}[n]$ as an element of the emotion vector $\mathbf{e}[n]$ that contains all emotion values if it is needed. The authors in this paper will soon publish a paper that explains how to formulate the previously exposed theories as a computational model to calculate a robot's emotional states.

With these evaluated emotions, a robot is able to show not only its emotional expressions but also reveal its internal motivation states in given situations, so that human companion can recognize robot's attention level and realize how much the situations are important to the robot.

\subsection{Behavior Selection based on Emotional Experiences}

While motivational emotions are being generated, the emotional experiences are also memorized with the situational information. Especially, events that cause highly aroused emotion are well remembered [17][18]. Then, the selectively remembered emotional experiences affect attention and selection preference when behavior decision is being made [19][20].

Likewise, a robot may store and use its emotional experiences. Since the robot's emotion elements, valence and arousal, are obtained from the motivational emotion generation model, the robot can selectively memorize events which are attentive based on the arousal level. Later when the robot faces situations, it can divide its attention proportional to emotional levels that are related to the situations. The higher level of emotion causes more attention to the event, and the robot has preferences to the decision choices that match attributes of emotional experiences. The integration of Emotional memory and behavior decision in an autonomous robot has been recently tried by researchers [21-25].

Suppose one sample $S_{k}$ consisting of situational states $\mathbf{s}\left[n_{k}\right]$, the robot's emotion $\mathbf{a}\left[n_{k}\right]$, and step $n_{k}$ is stored when an emotion is evoked at step $n_{k}$. Then, the set of all samples $\mathbf{S}$ can be described as 


$$
\mathbf{S}=\left\{S_{k} \mid S_{k}=\left(\mathbf{s}\left[n_{k}\right], \mathbf{a}\left[n_{k}\right], n_{k}\right), k=1 \cdots N\right\}
$$

where $N$ is the number of samples. If a situation is given as $\mathbf{s}_{\text {given }}$ in the recalling stage, corresponding subset of samples is described as

$$
\mathbf{S}^{\prime}=\left\{S_{k} \mid S_{k}=\left(\mathbf{s}_{\text {given }}, \mathbf{a}\left[n_{k}\right], n_{k}\right), S_{k} \in \mathbf{S}\right\}
$$

As explained above, the level of arousal is used as modulating factor for memory. The modulating factor $\alpha_{k}$ can be redefined as (5) within a range $[0,1]$ and the modulated and re-evaluated valence $v_{k}[n]$ of one sample at step $n$ can be defined as (6)

$$
\begin{gathered}
\alpha_{k}=\left(\operatorname{arousal}\left[n_{k}\right]+1\right) / 2 \\
v_{k}[n]=\alpha_{k} \cdot \operatorname{valence}\left[n_{k}\right] \cdot \gamma\left(n-n_{k}\right)
\end{gathered}
$$

where $\gamma(\cdot)$ is a time regression function with which more recent emotional experiences remain significant. Then, given the situation, the total accumulated valence $v_{\text {total }}[n]$ among $\mathbf{S}^{\prime}$ at step $n$ can be evaluated by calculating

$$
v_{\text {total }}[n]=\left(\sum_{\mathbf{s}^{\prime}} v_{k}[n]\right) / M
$$

where $M$ is the normalization value which can be calculated as $\sum_{\mathbf{s}^{\prime}} \gamma\left(n-n_{k}\right)$.

Now, suppose the set of robot's action candidates for the situation is $\mathbf{A}$ and each action candidate has valence attribute $v_{\text {action }}$, for example, giving the glad hand is an action with positive valence and avoiding is an action with negative valence. Then, the robot makes a decision: selecting an action that matches emotional experiences.

$$
\text { action }=\arg \min _{\mathrm{A}}\left|v_{\text {action }}-v_{\text {total }}\right|
$$

\section{Experimental Application Scenario}

For the experiment, authors of this paper have implemented a joint attention scenario in which a human and a robot play a 2048 game together shown as in Fig 1. Basic situation is that the robot recommends a direction at every step of the game and the human participant press a key to move to next step in which the participant doesn't need to follow the robot's recommendation. According to the participant's reaction, the robot generates its motivational emotion, and shows its emotional expressions. During that time the experienced emotion is simultaneously memorized with the user information. The game ends when the score reaches to 2048 or when there is no possible direction.

In this joint attention scenario, the robot has distinguished motivational states. For the value effectiveness, the robot is motivated to get 2048 score or higher score of the game than previous game scores. For the truth effectiveness, the robot is motivated to establish consistency of the acceptance rate of the human participant. For the control effectiveness, the robot is motivated to make the human follow its recommendation so that it can manage the game. These motivational states may differ between robots' personalities or developer's design purposes. 

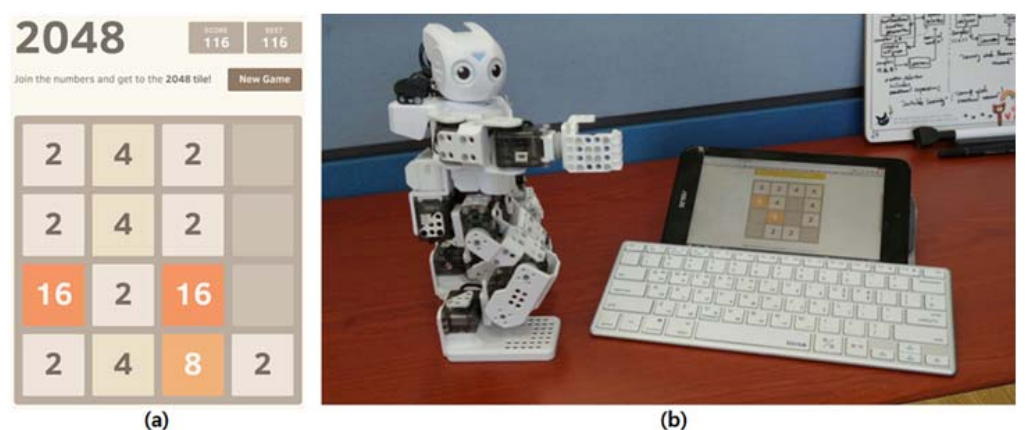

Fig. 1. (a) 2048 game implemented in MATLAB; (b) 2048 game is displayed on a window pad and DARWIN-MINI is recommending a direction for next step of the game.

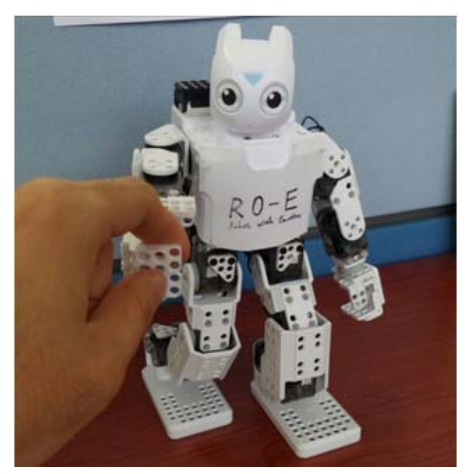

Fig. 2. An interaction scenario after 2048 game in which the robot decides whether to follow human's lead or not

After the game ended, another interaction scenario is situated in which the human leads the robot to go somewhere by pulling the robot's hand as shown in Fig. 6, then the robot decides whether to follow human's lead or not in accordance with the robot's emotional experiences which were previously generated and memorized through previous game.

\section{$4 \quad$ Preliminary Result and Discussion}

A preliminary pilot test was conducted and examined if the proposed method would perform properly as intended. Figures from Fig. 3 to Fig. 7 present the preliminary result.

Fig. 3 shows an example of changes of the game score and acceptance rate from step 1 to step 300. The game score is the biggest block number of the board, and acceptance rate is obtained from the average rate of acceptancy in recent 30 steps. As it is shown, the acceptance rate is not consistent in time and it depends on how much the human user has intention to follow the robot's recommendation. 
Fig. 4 shows the corresponding changes of three types of effectiveness: value, truth, and control. 1 denotes success of effectiveness and -1 denotes failure of effectiveness. Value effectiveness is sensitive to the game score as shown Fig. 4 (a). If the game score increases, i.e., the biggest block changes to higher number, the robot gets closer to the goal and the value effectiveness is successful in the progress. Otherwise, if the game score doesn't increase, the likelihood to the ending condition of the game at which there is no direction to move blocks is getting higher, so that the value effectiveness is failed. Truth effectiveness depends on the consistency of acceptance rate as shown Fig. 4 (b). If consistency of acceptance rate of human is high, the robot can establish the reality of how frequently human will follow the robot's recommendation or not, so the truth effectiveness is successful. Otherwise, the truth effectiveness is failed. Control effectiveness is sensitive to the acceptance rate by smoothing the acceptance rate as shown in Fig. 4 (c). If the acceptance rate is higher than 0.5, then the robot has more authority to manage the game and the control effectiveness is successful. If the acceptance rate is lower than 0.5 , the robot has lower authority so that the control effectiveness is failed.

Fig. 5 shows valence and arousal factor of robot's emotion corresponding to successes or failures of effectiveness. Valence can be directly evaluated by averaging three types of effectiveness. In Fig. 5 (b), changes of the level of arousal are depicted with each portion of each type of effectiveness. Though in this paper, the portion of each type of effectiveness is averaged, it could be weighted by predefined personality or could follow the winner-takes-all rule, or could affect emotion independently.

Fig. 6 shows specific emotions $e_{i}[n]$ which is independently calculated by equation (2). For simplicity, in this paper only 8 emotions are depicted in Fig. 6, which emotions are the representative of each quadrat or axis of two-dimensional emotion space and picked from Circumplex model. First four emotions: pleased, exited, aroused, and distressed depicted in Fig. 6 (a), are more dominant emotions, Later four emotions: miserable, depressed, sleepy, and content depicted in Fig. 6 (b), are less dominant emotions in this result. During the first 50 steps, aroused and distressed emotions were dominantly generated because there was no significant reward from game and lots of uncertainty of the game. Exited and aroused emotions were dominantly generated near 100-th step because there was a reward that is from score increase and consistent acceptance rate. During the last 80 steps, exited and aroused emotion were dominantly generated because there were a big score change and consistent acceptance rate.

As mentioned in chapter 3, after 2048 game ended, an additional interaction scenario happened. The human leads the robot to go somewhere by pulling the robot's hand, then the robot decides whether to follow human's lead or not. In the interaction scenario, there were four action candidates for the robot: shaking off, ignoring, hesitating, carefully following, and openly following with valence attribute $-1,-0.5,0$, 0.5 , and 1 , respectively.

Assuming that the 2048 game ended at 300th step, the total accumulated valence $v_{\text {total }}$ at 300th step was about 0.1 as shown in Fig. 7. As looking at the changes of the total accumulated valence $v_{\text {total }}$, it was negative during the first quarter of steps, and 


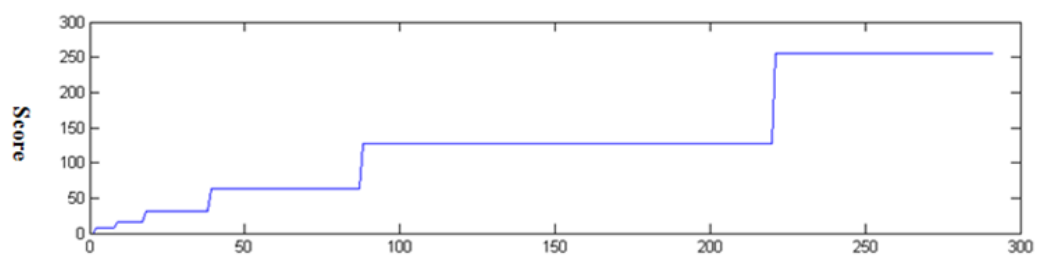

(a)

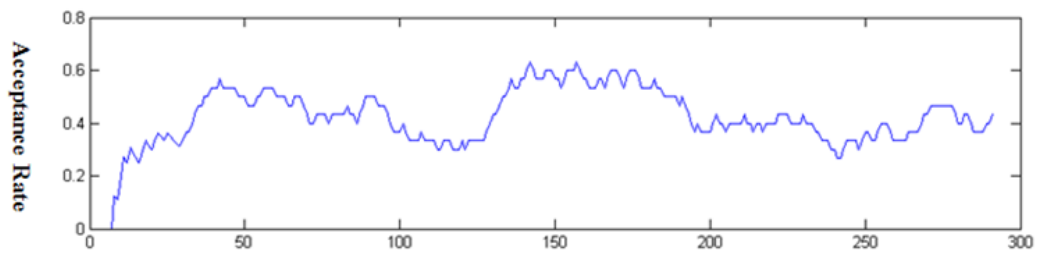

(b)

Fig. 3. (a) Changes of the game score which is the biggest number of the game board; (b) Average acceptance rate within recent 30 steps
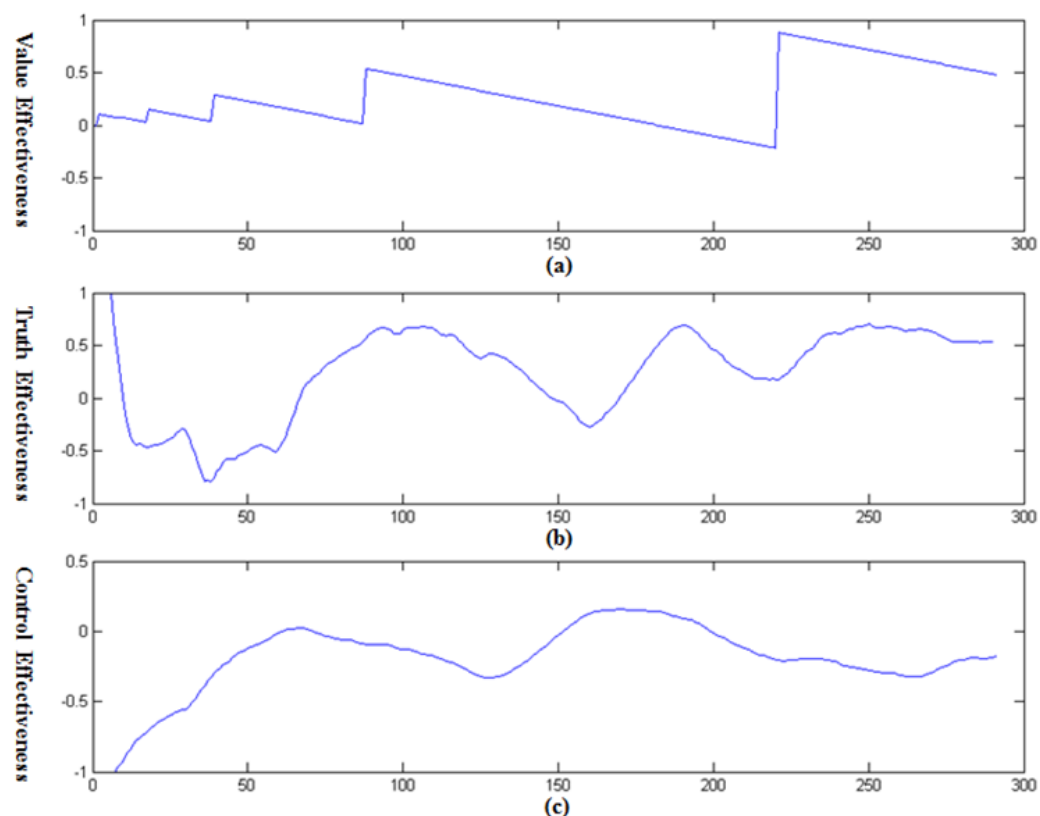

Fig. 4. (a) Changes of value effectiveness; (b) Changes of truth effectiveness; (c) Changes of control effectiveness 


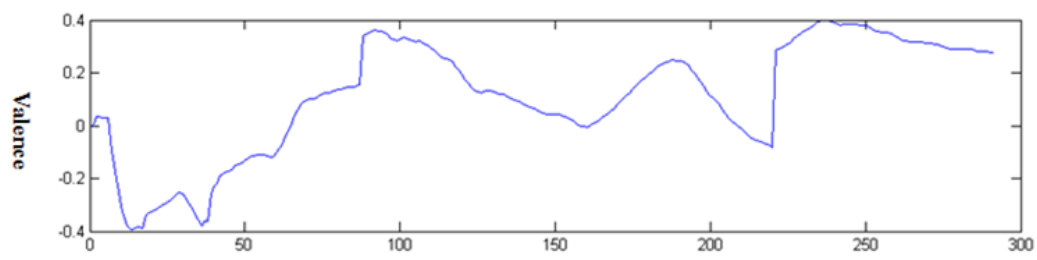

(a)

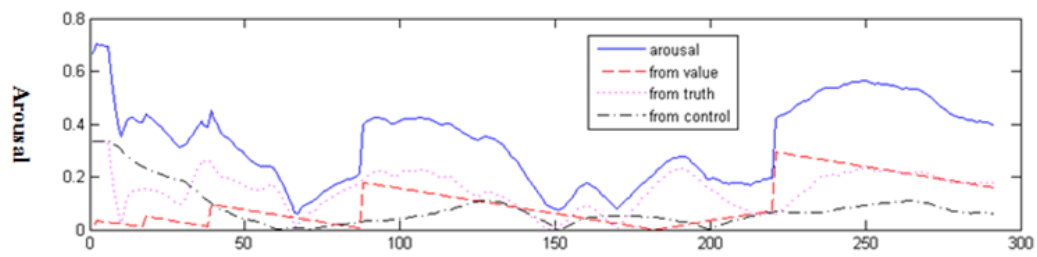

(b)

Fig. 5. (a) Changes of valence;

(b) Changes of arousal depicted with each portion of effectiveness

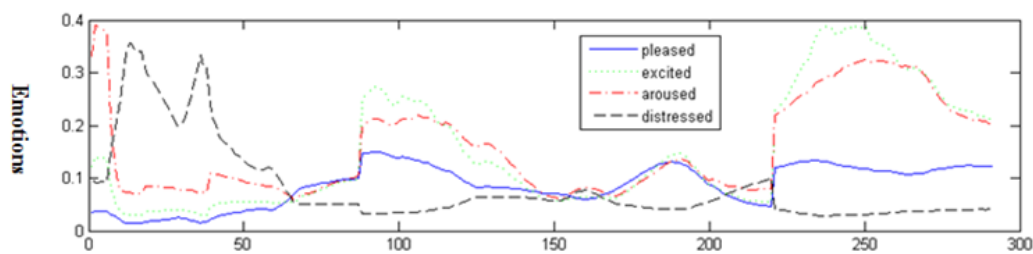

(a)

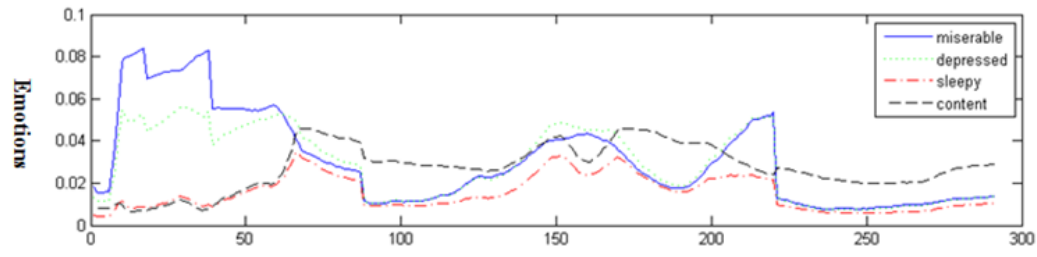

(b)

Fig. 6. Intensity changes of each emotion (depicted separately for readability)

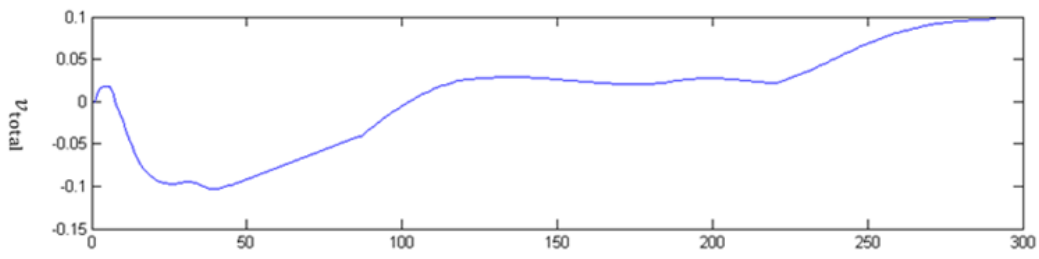

Fig. 7. Changes of the total accumulated valence $v_{\text {total }}$ 
during the middle period, the valence value was recovered, and then finally $v_{\text {total }}$ reached almost to the number 0.1 although this number is not significantly big.

At the behavior selection process, the robot decided an action which has the closest valence attribute, i.e. to hesitate to follow the human's lead, which means that the robot still doesn't think the human is friendly enough. The robot may sometimes carefully follow the human's lead with this $v_{\text {total }}$ by adding probabilistic parameter to the decision process. This probabilistic concept will be discussed in next paper.

The reason why the value is not big enough is that the subject didn't follow the robot's recommendation very much. Maybe the subject was motivated to play the game just for enjoying himself/herself. For next experiments, it seems to be needed to give reward to subjects so that they are motivated to get higher score and more to follow the robot's recommendation, then, compare results caused by them to results caused by those who are not motivated to follow the robot's recommendation.

For further study, two kinds of robots will be compared: one robot acting by the proposed method, and the other acting randomly. Then, we will monitor participants' psychological changes such as how the participants feel when the robot shows emotional expressions while playing the game, how the participants feel when the robot follows or disobey participants' lead after the game, how much the participants' acceptance rate of robot's recommendation changes when playing more games.

The authors of this paper are expecting the participants to anthropomorphize the robot that has motivational emotions and emotional experiences and to think that the robot has a personality; the acceptance rate or robot's recommendation will increase; and finally participants will accept the robot as a social partner.

\section{Conclusion}

In this paper, a motivational emotion generation model is proposed. The model employs the unified motivation theory which is proposed by Higgins, and explains relations between motivation and emotion. In addition, a behavior selection model based on emotional experience is suggested; with which decision preferences are more affected by highly aroused and attentive emotional memory.

To test the proposed method, a joint attention scenario is suggested in which a human and a robot play a 2048 game together and a follow-up interaction is conducted to observe the robot's behavior selection that is influenced through the game. A preliminary pilot test was conducted and examined if the proposed method would perform properly as intended. As a result, the robot showed corresponding motivational emotions and behaved properly based on the emotional experiences in the proposed framework. However, more advanced experiments are needed, and further plans and expectations are discussed for a comparison of a robot that has motivational emotion and emotional experiences and another robot which is not.

Acknowledgement. This work is supported by the Industrial Strategic Technology Development Program (10044009) funded by the Ministry of Trade, Industry and Energy (MOTIE, Korea) 


\section{References}

1. Lin, Patrick, Keith Abney, and George A. Bekey. Robot Ethics: The Ethical and Social Implications of Robotics. The MIT Press, 2011

2. Scheutz, Matthias. "What Is Robot Ethics?[TC Spotlight]" Robotics \& Automation Magazine, IEEE 20.4 (2013): 20-165.

3. Breazeal, Cynthia, "Function meets style: insights from emotion theory applied to HRI,”Systems, Man, and Cybernetics, Part C: Applications and Reviews, IEEE Transactions on 34.2, pp. 187-194, 2004.

4. Arkin, Ronald C., et al. "An ethological and emotional basis for human-robot interaction." Robotics and Autonomous Systems 42.3 (2003): 191-201.

5. Zecca, Massimiliano, et al. "Whole body emotion expressions for KOBIAN humanoid robot-preliminary experiments with different Emotional patterns-." Robot and Human Interactive Communication, 2009. RO-MAN 2009. The 18th IEEE International Symposium on. IEEE, 2009.

6. Kaneko, Kenji, et al. "Cybernetic human HRP-4C." Humanoid Robots, 2009. Humanoids 2009. 9th IEEE-RAS International Conference on. IEEE, 2009.

7. Yuk, Nam-Su, and Dong-Soo Kwon. "Realization of expressive body motion using legwheel hybrid mobile robot: KaMERo1." Control, Automation and Systems, 2008. ICCAS 2008. International Conference on. IEEE, 2008.

8. Miwa, Hiroyasu, et al. "A new mental model for humanoid robots for human friendly communication introduction of learning system, mood vector and second order equations of emotion,” Robotics and Automation, 2003. Proceedings. ICRA'03. IEEE International Conference on. vol. 3. IEEE, 2003.

9. Kwon, D.S., "Emotion interaction system for a service robot", 16th IEEE International Conference on Robot \& Human Interactive Communication, 2007

10. Lee, Won Hyong, et al. "Robot's emotion generation model for transition and diversity using energy, entropy, and homeostasis concepts,” Robotics and Biomimetics (ROBIO), 2010 IEEE International Conference on. IEEE, 2010.

11. Lang, Peter J., Margaret M. Bradley, and Bruce N. Cuthbert. "Motivated attention: Affect, activation, and action." Attention and orienting: Sensory and motivational processes (1997): 97-135.

12. Higgins, E. Tory. "Promotion and prevention experiences: Relating emotions to nonemotional motivational states." Handbook of affect and social cognition (2001): 186-211.

13. Plutchik, Robert. "A psychoevolutionary theory of emotions." Social Science Information/sur les sciences sociales (1982).

14. Kalat, James, and Michelle Shiota. Emotion. Cengage Learning, 2011.

15. Higgins, E. Tory. Beyond pleasure and pain: How motivation works. Oxford University Press, 2011.

16. Russell, James A., "A circumplex model of affect," Journal of Personality and Social Psychology, Vol 39(6), Dec 1980

17. McGaugh, James L. "Memory--a century of consolidation." Science 287.5451 (2000): 248251.

18. Phelps, Elizabeth A. "Human emotion and memory: interactions of the amygdala and hippocampal complex." Current opinion in neurobiology 14.2 (2004): 198-202.

19. G. Loewenstein and J. Lerner, "The role of affect in decision making," in Handbook of Affective Science, R. Davidson, H. Goldsmith, and K. Scherer, Eds. Oxford, NY, USA: Oxford University Press, 2003, pp. 619-642. 
20. E. Peters, "The functions of affect in the construction of preferences,"in The construction of preference, S. Lichtenstein and P. Slovic, Eds. New York, NY, US: Cambridge University Press, 2006, pp. 454-463.

21. Novianto, Rony, and Mary-Anne Williams. "Innate and Learned Emotion Network." BICA. 2011.

22. M. B. Moussa and N. Magnenat-Thalmann, "Toward socially responsible agents: integrating attachment and learning in emotional decisionmaking, " Computer Animation and Virtual Worlds, vol. 24, no. 3-4, pp. 327-334, 2013.

23. Novianto, Rony, Benjamin Johnston, and Mary-Anne Williams. "Habituation and Sensitisation Learning in ASMO Cognitive Architecture." Social Robotics. Springer International Publishing, 2013. 249-259.

24. Ruby Valverde Ibánez, Matthias U. Keysermann and Patr'ıcia A. Vargas, "Emotional Memories in Autonomous Robots," IEEE International Symposium on Robot and Human Interactive Communication(RO-MAN) 2014

25. Johannes Feldmaier, and Klaus Diepold, "Path-finding Using Reinforcement Learning and Affective States," IEEE International Symposium on Robot and Human Interactive Communication(RO-MAN) 2014 